\title{
Le vent des globes
}

\section{Julia Vecsey}

Dr méd., membre de la FMH

Ils étaient 29 voiliers à prendre le départ de la course mythique du Vendée Globe, course autour du globe terrestre avec sur chaque bateau un seul skipper.

La course commence dans l'Atlantique, mais dès le passage de l'équateur, commencent les choses vraiment sérieuses. La température est encore agréable à cette latitude, mais ça ne va pas durer...

Profitant encore de la relative tranquillité des éléments, les marins peuvent écouter les informations, et apprendre ainsi que la victoire de l'oncle Donald n'est ni une blague ni une trumperie, ils croisent des drones chargés de médicaments qui seront livrés à des malades isolés sur le continent africain.

L'Antarctique approche, avec son cortège d'icebergs magiques et menaçants, sa mer déchaînée, et les paquets d'eau qui se déversent contre les coques mettent les navires à rude épreuve. Cependant il n'y a pas que l'eau qui agresse les bateaux... la mer sert de dévaloir à certains cargos, et on croise à la surface des océans toutes sortes de déchets agressifs responsables de dégâts catastrophiques sur tout ce qu'il rencontrent, provoquant des plaies définitives sur les esquifs.

Autre pollution des océans, le bruit sous-marin sème la confusion au sein de la faune, qui est parfois totalement désorientée: c'est ainsi qu'un dauphin, égaré dans une région inhabituelle pour lui, avait croisé Roland Bourdougnon, autre vaillant navigateur, perdu au fond de l'océan lors d'une plongée sous-marine bien imprudente, en solitaire. Roland n'a trouvé le salut que grâce à ce dauphin en perdition, croisé avec bonheur alors que sa bonbonne d'oxygène fournissait ses dernières bulles. Le plongeur exténué, qui ne pouvait que flipper dans cette situation désespérée, s'est agrippé à l'aileron du sympathique mammifère compréhensif qui l'a ramené vers la surface, lui sauvant ainsi la vie. Pourtant la partie n'était pas encore gagnée pour Roland, qui certes se retrouvait agrippé à l'aileron de son hôte à l'air libre, mais qui devait manger et boire pour continuer à vivre! La complicité établie avec le dauphin permit à notre marin de partager le fruit de la pêche du cétacé, et ainsi de combler quelque peu son creux. Mais il fallait encore boire, les jours passaient... et comme la vie est décidément pleine de surprises, c'est alors que... Roland vit se profiler à l'horizon la silhouette d'un voilier, géant des mers, qui participait à la course.

Le skipper de ce bateau, candidat à la victoire, très vigilant à la barre, scrutait l'horizon autour de lui quand il aperçut le dauphin et son étrange cavalier! Il s'empara de ses jumelles, et vit qu'il s'agissait d'un homme! Il se dérouta, et se dirigea vers le couple étrange. Quand il fut plus proche de lui, il reconnut Roland, vieux pote qu'il croyait mort, et en perdit presque pied!

Il fit monter le naufragé à bord, et Roland, après des adieux émus au dauphin, raconta comment celui-ci lui avait offert un bol d'air salvateur. Pendant ce temps, le cétacé les accompagnait, batifolant joyeusement tout autour du bateau.

Le règlement de la course est formel: le pilote doit être seul! Qu'à cela ne tienne, sur ce bateau-là, ils seraient désormais deux, quitte à encourir la disqualification! Un problème logistique se posa alors: il n'y avait de la nourriture que pour un seul homme à bord! Et la route était longue encore jusqu'à bon port. C'est alors que la direction de la course, dûment informée du miracle de ce sauvetage, décida de dépêcher un drone chargé de nourriture lyophilisée vers la bête de course, un drone de la même espèce que ceux envoyés en Afrique..

La question qui se posait aux deux navigateurs était simple: abandonner le tour du monde et rebrousser chemin, ou poursuivre dans la même direction? La moitié du parcours était couverte, et faire demi-tour impliquait de faire face à des vents défavorables. La décision fut donc prise de continuer la route.

Avec deux marins à bord, les manœuvres étaient beaucoup plus rapides, plus efficaces, la gestion du sommeil moins contraignante, même le passage du cap Horn ne fut qu'une formalité; nos deux navigateurs gagnèrent ainsi du terrain, et furent les premiers à retourner en Vendée.

Même s'ils étaient maintenant hors course, la foule amassée dans l'aire d'arrivée les accueillit en grande pompe, et fit un triomphe aux deux aventuriers.

Personne ne remarqua un dauphin qui les talonnait joyeusement... 